\title{
A Formal Model of Clock Domain Crossing and Automated Verification of Time-Triggered Hardware
}

\author{
Julien Schmaltz ${ }^{1}$ \\ Institute for Computing and Information Sciences \\ Radboud University Nijmegen, The Netherlands \\ Email: julien@cs.ru.nl
}

\begin{abstract}
We develop formal arguments about a bit clock synchronization mechanism for time-triggered hardware. The architecture is inspired by the FlexRay standard and described at the gate-level. The synchronization algorithm relies on a specific value of a counter. We prove or disprove values proposed in the literature. Our framework is based on a general and precise model of clock domain crossing, which considers metastability and clock imperfections. Our approach combines this model with the state transition representation of hardware. The result is a clear separation of analog and digital behaviors. Analog considerations are formalized in the logic of the Isabelle/HOL theorem prover. Digital arguments are discharged using the NuSMV model checker. To the best of our knowledge, this is the first verification effort tackling asynchronous transmissions at the gate-level.
\end{abstract}

\section{INTRODUCTION}

Embedded systems comprise software applications, compilers, real-time operating systems, processors with memory management units and devices, as well as communication architectures. These different components form the layers of a stack. The top layer and the most abstract one is occupied by software applications. Going down in the abstraction, software applications together with an operating system are compiled into machine code and run on top of processing units and memories. Their gate level description constitutes the lowest layer of the stack. In the late 80's, Bevier et al. [2] demonstrated a first "stack proof", i.e. a proof of a simulation theorem between the top layer and the bottom layer. The application of this approach to realistic embedded systems remains a challenge [12]. Computer systems are often distributed. One verified stack is not enough. One needs to prove correctness of stacks and their communications.

These communications are inherently asynchronous as in practice clocks of interconnected devices are not constant over time. This clock distortion induces possible metastable states of registers. The proof of distributed stacks requires the analysis of these phenomena at the gate-level. Moreover, in the context of realistic worst case execution time analysis, it is also necessary to know the duration of the transmission. Towards this end, a pencil and paper proof of an entire distributed systems was developed [1]. From this study, we formalized in

\footnotetext{
${ }^{1}$ Part of this work was carried out while the author was affiliated with the University of Saarland, Saarbrücken, Germany. This work was funded by the German Federal Ministry of Education and Research $(b m b+f)$ in the framework of the Verisoft project under grant 01 IS C38.
}

the logic of Isabelle/HOL [13] the bit transmission between independently clocked registers, assuming precise timing parameters and metastability [15].

The contribution of this paper is an important extension of these theoretical results and the definition and the application of a methodology for the verification of time-triggered hardware. We extend the Isabelle theory to allow for longterm jitter. This relieves the previous hypothesis about constant clock periods. The outcome is a general model of clock domain crossing. This model is combined with the semantics of transition systems used to describe hardware designs. This identifies constraints on the digital design that guarantee proper transmission assuming analog behaviors. These constraints can be solved by decision procedures. We use the integration in Isabelle of the model checker NuSMV [16]. We demonstrate this methodology on the verification of a timetriggered bus interface inspired by the FlexRay standard [5] and described at the gate-level. The statement of our theorem also includes the duration of the transmission. Our analysis identifies precisely the possible values of one crucial parameter of the bit clock synchronization mechanism. This proves and disproves values proposed in the literature.

The paper is structured as follows. In the next Section, we present our general model of clock domain crossing. We show in Section III how we use this model for hardware design verification. Section IV describes the time-triggered interface, which is verified in Section V. Related work is discussed in Section VI. Section VII concludes the paper.

\section{A Formal Model of Clock Domain Crossing}

\section{A. Signals and Clocks}

Time is represented by the nonnegative reals $\left(R_{\geq 0}\right)$. A signal $s$ is represented by a function $s(t)$ from real time $t$ to $\{0,1, \Omega\}: 1$ and 0 mean "high" and "low" voltages; $\Omega$ means any voltage.

The clock period of unit $u$ is noted $\tau_{u}$. Periods are different from zero. The date of the $c^{\text {th }}$ rising edge of clock $c l k_{u}$ of unit $u$ is noted $e_{u}(c)$. It equals the product of $c$ with the clock period: $e_{u}(c)=c \cdot \tau_{u}$.

Function $e$ gives the ideal date of edges. In practice, it is impossible to guarantee constant clock periods. We assume that all clock periods of any clock deviate at most by a percentage $\delta$ of a reference clock period. This reference clock 


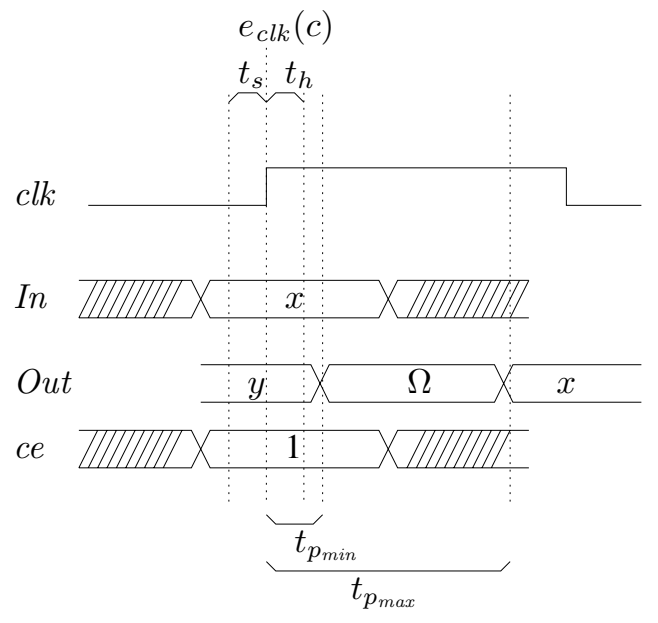

Fig. 1. Behavior of the register w.r.t clock edge $c$

is named $c l k_{r e f}$. Its period is $\tau_{r e f}$. Formally, we assume:

$$
\Gamma_{u} \equiv 1-\delta \leq \frac{\tau_{u}}{\tau_{\text {ref }}} \leq 1+\delta
$$

We are not interested in the deviation at each cycle, but in the number of cycles in which the number of ticks of two independent clocks may differ by at most one. Let $\pi$ be that number. In this interval, the maximum drift between two clocks is obtained between the slowest and the fastest clocks allowed by Equation 1. Consequently, the ratio between the minimum and the maximum clock periods defines the lower bound of the drift. From Equation 1 and defining $\pi=\frac{1-\delta}{2 \cdot \delta}$, we prove the following lemma:

\section{Lemma 1: Bounded Clock Drift.}

$$
\Gamma_{i} \wedge \Gamma_{j} \rightarrow \frac{\pi}{\pi+1} \leq \frac{\operatorname{Min}\left(\tau_{i}, \tau_{j}\right)}{\operatorname{Max}\left(\tau_{i}, \tau_{j}\right)}
$$

This property is preserved for any number less than $\pi$.

\section{B. Analog Registers}

Open intervals are represented using open squared brackets. Shifting an interval is noted $x+[y: z]$ instead of $[x+y: x+z]$.

Registers consist of one input signal $I n$, one clock signal $c l k$, one control signal $c e$ and one output signal Out (Fig. 1). A new value $(x)$ is input to the register at cycle $c$, which is defined by interval $\left[e_{u}(c): e_{u}(c+1)[\right.$. During minimum propagation delay $t_{p_{\min }}$ the output signal equals previous value $y$. Because the control signal is high, the output oscillates (i.e. is $\Omega$ ) before stabilizing at new value $x$. If the control signal is low, the output does not oscillate and keeps its old value $y$.

If the input or the control signals do not have a constant value during the setup time (noted $t_{s}$ ) before edge $c$ and during the holding time (noted $t_{h}$ ) after edge $c$, the register may become metastable. This means that its output may still be $\Omega$ after $t_{p_{\max }}$. When this metastable state is resolved, the register reaches a defined value. Metastability cannot be avoided [9]. We assume that this resolution time is less than one clock period. Before giving our formal definition of analog registers, we define a few concepts.
${ }_{a} R_{u}\left(c, c l k_{u}, c e_{u}, \operatorname{In}_{u}, O u t_{u}^{0}\right) \triangleq$

$$
\begin{aligned}
& \text { if } c=0 \text { then } \lambda t \text {.Out } t_{u}^{0} \text { else } \\
& \text { if }\left\{\begin{array}{r}
\operatorname{stadep}\left(e_{u}(c)-t_{s}, e_{u}(c)+t_{h}, c e_{u}\right) \\
\wedge \operatorname{stadep}\left(e_{u}(c)-t_{s}, e_{u}(c)+t_{h}, I n_{u}\right)
\end{array}\right. \text { then } \\
& \text { if } c e_{u}\left(e_{u}(c)\right)=1 \text { then } \\
& \lambda t . \begin{cases}{ }_{a} R_{u}(c-1, \ldots)\left(e_{u}(c)\right) & \left.\left.: t \in e_{u}(c)+\right] 0: t_{p_{\min }}\right] \\
\Omega & \left.: t \in e_{u}(c)+\right] t_{p_{\min }}: t_{p_{\max }}[ \\
\operatorname{In}_{u}\left(e_{u}(c)\right) & : t \in e_{u}(c)+\left[t_{p_{\max }}: \tau_{u}\right] \\
\Omega & \left.: t \notin] e_{u}(c): e_{u}(c+1)\right]\end{cases} \\
& \text { else ;; keep old value } \\
& \lambda t .\left\{\begin{array}{lll}
{ }_{a} R_{u}(c-1, \ldots)\left(e_{u}(c)\right) & : & \left.\forall t \in] e_{u}(c): e_{u}(c+1)\right] \\
\Omega & : & \left.t \notin] e_{u}(c): e_{u}(c+1)\right]
\end{array}\right. \\
& \text { endif } \\
& \text { else ;; metastability } \\
& \lambda t . \begin{cases}{ }_{a} R_{u}(c-1, \ldots)\left(e_{u}(c)\right) & \left.\left.: t \in e_{u}(c)+\right] 0: t_{p_{\text {min }}}\right] \\
\Omega & \left.: t \in e_{u}(c)+\right] t_{p_{\text {min }}}: \tau_{u}[ \\
x \in\{0,1\} & : t=e_{u}(c+1) \\
\Omega & \left.: t \notin] e_{u}(c): e_{u}(c+1)\right]\end{cases} \\
& \text { endif }
\end{aligned}
$$

Fig. 2. Definition of Analog Registers

The metastability window w.r.t. edge $c$ of register $u$ (noted $\left.\mathrm{MW}_{u}^{c}\right)$ is defined by interval $e_{u}(c)+\left[-t_{s}: t_{h}\right]$.

A signal $s$ is stable during time interval $\left[t_{1}: t_{2}\right]$ if it holds the value at time $t_{1}$ until time $t_{2}$. A signal $s$ has a defined value during time interval $\left[t_{1}: t_{2}\right]$ if it never equals $\Omega$ during that interval. Formally, this is expressed as follows ${ }^{1}$ :

$$
\operatorname{stadep}\left(t_{1}, t_{2}, s\right) \triangleq \exists b \in\{0,1\}, \forall t \in\left[t_{1}: t_{2}\right], s(t)=b
$$

The formal definition of the analog behavior is given by function ${ }_{a} R_{u}$ (Fig 2). We are interested in the output value of a register for all real times during cycle $c$. Function ${ }_{a} R_{u}$ takes as arguments a cycle $c$, a clock signal $c l k_{u}$, a clock enable control signal $c e_{u}$, an input signal $I n_{u}$, and the initial output value $O u t_{u}^{0}$. It generates a signal.

If no setup or holding time violation occurs, the register behaves normally. If the control signal is low, the register keeps its old value (at the previous cycle $c-1$ ); if the control signal is high the output keeps its previous value during $t_{p_{\min }}$, then oscillates (i.e. is $\Omega$ ) to finally reach its final value at time $e_{u}(c)+t_{p_{\max }}$. If input signal $I n_{u}$ or control signal $c e_{u}$ is not stable and defined during the metastability window, the register becomes metastable. The output equals the previous computation until $t_{p_{\min }}$ (included) and $\Omega$ afterwards. At the end of the cycle, metastability has been resolved and the output equals an arbitrary but defined value. To make the function total, $\Omega$ is output for all times outside the cycle. To alleviate our notation, we shall write ${ }_{a} R_{u}^{c}$ instead of ${ }_{a} R_{u}\left(c, c l k_{u}, c e_{u}, \operatorname{In}_{u}, O u t_{u}^{0}\right)$.

Formally, all timing parameters $\left(t_{h}, t_{s}, t_{p_{\text {min }}}, t_{p_{\text {max }}}\right)$ are percentages. We assume that their sum is less than 1 . Their value depends on the local clock period. In the remainder of this

\footnotetext{
${ }^{1}$ Note: stadep means stable, defined, predicate
} 
functions. Before describing our approach, we define type conversion functions and rephrase Theorem 3 to match bits and not signals.

\section{A. Type Conversions}

The conversion from bit lists to signals is done by function $\gamma$. We do not give a particular definition to this function. We only assume that it produces a signal such that during the metastability window around cycle $i+1$, it outputs the value with index $i$ in the bit list. This property is defined by predicate bv2sp:

$$
\operatorname{bv2sp}\left(\gamma, l_{u}\right) \equiv \forall t, i, t \in \mathrm{MW}_{u}^{i+1} \rightarrow \gamma\left(l_{u}\right)=l_{u}[i]
$$

The conversion from signals to bits is done by function $\zeta$, which takes as input a signal and a time. If the value of the signal at that time is a bit value, then this value is returned. Otherwise, some bit value is returned.

$$
\zeta(s, t) \triangleq \text { if } s(t) \in\{0,1\} \text { then } s(t) \text { else } x \in\{0,1\}
$$

\section{B. Transfer Correctness in the Digital World}

Let lists $c e_{s}$ and $I_{s}$ be the bit lists containing values given to the analog sender register. If they both satisfy predicate bv2sp, list element $c e_{s}[c-1]$ or $\operatorname{In}_{s}[c-1]$ corresponds to the bit value given to the sender analog register at time $e_{s}(c)$.

Theorem 3 is embedded into a digital context in the following statement. We assume that (a) clock drift is bounded; (b) function $\gamma$ correctly translates bit lists $c e_{s}$ and $\mathrm{In}_{s}$; (c) the digital control bits are high once and then low $k$ times. Analog hypotheses are concerned with the connection of the sender with the receiver and the clock drift. Obviously, they cannot be "digitalized". Under these assumptions, we prove that the "digitalized" output of the analog receiver register equals the digital input of the sender at cycle $c$. In the remainder of this paper, we will denote the conjunction of the hypotheses of this theorem by $\mathcal{H}$.

\section{Theorem 4: Back to the Digital World.}

$$
\begin{aligned}
& \Gamma_{r} \wedge \Gamma_{s} \wedge n+1 \leq k \leq \pi \wedge c y(\xi, c) \\
& \text { (*bounded drift, cy(c)*) } \\
& \wedge \quad \forall c, I_{r}={ }_{a} R_{s}\left(c, c l k_{s}, \gamma\left(c e_{s}\right), \gamma\left(\text { In }_{s}\right), \text { Out }_{s}^{0}\right) \\
& \wedge \forall t, c e_{r}(t)=1 \text { (*analog link*) } \\
& \wedge \operatorname{bv2sp}\left(\gamma, c e_{s}, c l k_{s}\right) \wedge \operatorname{bv2sp}\left(\gamma, \operatorname{In}_{s}, c l k_{s}\right) \\
& \text { (*modeling hypotheses*) } \\
& \wedge \quad c e_{s}[c+\alpha-1]=1 \wedge c>0 \\
& \left.\wedge \quad \forall l \in[1: k], c e_{s}[c+l-1]=0 \text { (*sender } \mathrm{OK}^{*}\right) \\
& \wedge e_{r}(\xi)+\left[-t_{s}: t_{h}\right] \in \mathrm{SSW}_{k}^{c}(* \operatorname{good} \text { cycle } *) \\
& \rightarrow \\
& \zeta\left({ }_{a} R_{r}^{\xi}, e_{r}(\xi+1)\right)=\operatorname{In}_{s}[c-1]
\end{aligned}
$$

Proof. By definition of predicate bv2sp, $\gamma\left(I n_{s}\right)$ and $\gamma\left(c e_{s}\right)$ are stadep for the required cycles. Theorem 3 concludes.

\section{Principle and Soundness}

Fig. 4 illustrates our integration of our analog results in the analysis of digital designs. Our clock domain crossing model (CDC) is shown inside the dashed box. The remainder of the figure corresponds to digital designs that are actually used

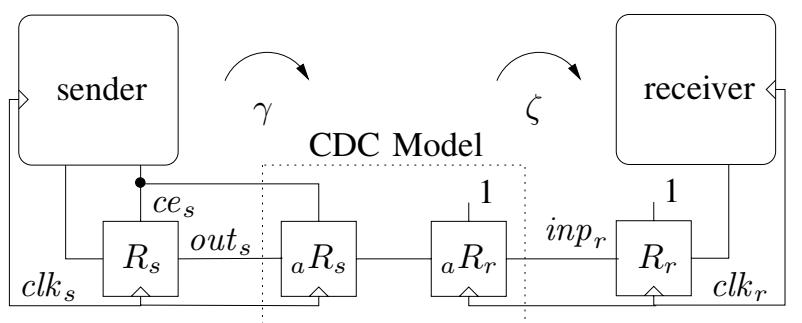

Fig. 4. Mixing Analog and Digital Signals

to synthesize hardware. These designs are not modified. Our model is simply inserted as a filter of the receiver inputs.

Digital designs are represented by their transition function, one application of which represents the computation of one clock cycle. The sender and the receiver parts are analyzed separately. The analysis of the sender does not need any analog arguments. It mainly consists of the proof that sender output out $_{s}$ follows a specific frame format. The analysis of the receiver is done assuming correctness of the sender and that the connection of receiver input $i n p_{r}$ is done through our CDC model. We write that an element $s_{u}$ of unit $u$ has bit-value $x$ at cycle $c$-i.e. after $c$ applications of the transition function - as $s_{u}^{c}=x$. Formally, we assume that the value of input bit $i n p_{r}$ at hardware cycle $c$ equals the output value of register ${ }_{a} R_{r}$ at the date of edge $c+1$ :

$$
\forall c, i n p_{r}^{c}=\zeta\left({ }_{a} R_{r}^{c}, e_{r}(c+1)\right)
$$

The left hand side represents the value that should be in register $R_{r}$ at $c+1$. As the analog register is not part of the transition function of the receiver, one application of the latter compensates this difference. The right hand side is always a defined value. This Equation only holds when $R_{r}$ is not metastable. As discussed in the next sub-section, it is always the case when we use Equation 2.

\section{Proof Method}

Our proof method uses Theorems 2, 1, 4 and Equation 2. We also need a mark $c y(\xi, c)$ which connects receiver cycle $\xi$ with the beginning of a safe sampling window started at sender cycle $c$. From this mark, we obtain from Theorem 2 that there are $n+1$ receiver edges in the safe sampling window. These edges may be shifted by one cycle depending on the resolution of metastable states. Then, we obtain from Theorem 4 that register ${ }_{a} R_{r}$ outputs out $t_{s}^{c}$ at the date of these edges. Because we are outside metastable behaviors, we obtain from Equation 2 inputs for the receiver. Once these inputs are known, the analysis is back to the digital world and decision procedures apply. We obtain subsequent inputs using a similar reasoning and the marks given by Theorem 1 .

\section{Time-Triggered Bus Interfaces}

We present an implementation of a time-triggered bus interface inspired by the FlexRay standard [5] for safetycritical automotive applications. This design has already been 
$c y(\xi, c) \quad c y(\xi+16+[-1: 1], c+16)$

$$
e_{s}^{\prime}(c) \text { TSS FSS }
$$

$\begin{array}{lllllllllllllllllllllllllll}\text { out }_{s} & 1 & 1 & 1 & 0 & 0 & 0 & 0 & 0 & 0 & 0 & 0 & 1 & 1 & 1 & 1 & 1 & 1 & 1 & 1 & 1 & 1 & 1 & 1 & 1 & 1 & 1\end{array}$

$i n p_{r}$

$$
\begin{aligned}
& \begin{array}{llllllllllllllllllllllll} 
& 0 & 0 & 0 & 0 & 0 & 0 & 0 & \mathbf{a} & 1 & 1 & 1 & 1 & 1 & 1 & 1 & 1 & 1 & 1 & 1 & 1 & 1 & 1 & 1
\end{array} \\
& \uparrow_{\beta_{c}^{\xi}}^{\xi}+16 \\
& z=\operatorname{BSS}[0] \wedge c n t=011 \\
& z=\operatorname{FSS} \wedge \text { cnt }=010
\end{aligned}
$$$$
\begin{aligned}
& \beta_{c}^{\xi}=0 \\
& \beta_{c}^{\xi}=1
\end{aligned}
$$

Fig. 7. Initial Transmission Phase

is known. The final statement is Theorem 5 below. The first line of the conclusion states that for all bytes we detect a mark for BSS[0]. The second lines states that at this cycle the control automaton could be in different states with different values of its counter. This different values come from previous bytes which may have suffered from clock drifts. The last lines state that under this uncertainty the automaton samples byte $\sharp i$ properly. This theorem also proves lower and upper bounds on the time at which the last byte is recovered. Using the mark of the conclusion, these bounds can be expressed as functions of the reference clock and the time $\left(e_{s}(c)\right)$ when the first bit is put on the bus by the sender.

\section{Theorem 5: Transmission Correctness.}

$$
\begin{aligned}
& \mathcal{H} \wedge z^{\xi}=\text { idle } \wedge c y(\xi, c) \\
& \forall c, i n p_{r}^{c}=\zeta\left({ }_{a} R_{r}^{c}, e_{r}(c+1)\right)\left(* \mathrm{~A} / \mathrm{D} \operatorname{mix}^{*}\right) \\
& \mathrm{WF}_{c e}\left(c e_{s}, L, k, c\right) \wedge \mathrm{WF}_{\text {In }}\left(\text { out }_{s}, L, c\right) \\
& \forall i<l, \quad \exists \nu, \quad c y(\nu, c+16+80 \cdot i)(* \operatorname{mark} *) \\
& \wedge \quad z^{\nu}=(\mathrm{FSS} \vee b[7]) \wedge c n t^{\nu}=(001 \vee 010) \\
& \vee \quad z^{\nu}=\operatorname{BSS}[0] \wedge c n t^{\nu}=(011 \vee 100) \\
& \begin{array}{ll}
\wedge & z^{\nu+78+w}=\mathrm{BSS}[0] \wedge c n t^{\nu+78+w}=010 \\
\wedge & \mathrm{BYTE}^{\nu+79+w}=\left\langle\mathrm{out}_{s}^{c+16+80 \cdot i+8 \cdot(j+2)}\right\rangle
\end{array} \\
& \text { where } w \in[0: 3] \text { and } j \in[7: 0]
\end{aligned}
$$

The proof is done by induction over $i$. For space reason, we only detail the base case, which is pictured in Fig. 7. The first two lines show the output of the sender and how it is seen by the receiver. Black boxes indicate possible metastability.

Because of clock drift, the BSS[0]-mark may appear on the receiver side 15,16 or 17 cycles after $\xi$. There is a potential metastability at cycle $\xi$. Depending on the value reached after resolution - that is depending on the value of $\beta_{c}^{\xi}$ - the receiver automaton reaches different state and counter values when the BSS[0]-mark is detected. In the figure, we show these values at $\beta_{c}^{\xi}+16$, where the automaton is either in state $\mathrm{BSS}[0]$ with a counter at 011 or in state FSS with a counter at 010 . In the following sections, we prove that the receiver recovers a byte for all these possibilities.

\section{Traversing Synchronization Edges}

Our reasoning is illustrated in Fig. 8. The first two lines show the output of the sender and how it is seen by the receiver. Black boxes indicate possible metastability. Question marks are used to denote unknown values.

We fix the initial step of the lemma to match the date of the detection of the BSS[0]-mark. We consider the case where the receiver is in state $\mathrm{BSS}[0]$ with a counter value at either 011 or 100.

According to Theorem 1 and assuming that the BSS[0]mark is known, the BSS[1]-mark has three possible dates. The potential metastability around that edge has the same three dates. We consider bits sampled by the receiver at these dates unknown. Another source of uncertainty resides in factor $\beta$. It is already represented by metastability. Therefore, at most three bits are unknown. Depending on the values of these three bits, the automaton will spend more or less time in the states of BSS. There is synchronization if the lower and the upper bound on this number of cycles allow proper sampling. This bounds are defined by the next lemma which imposes that the automaton reaches state $b[0]$ with counter value 011 in at least 15 , and at most 18 cycles.

Let $t$ be the date of the affected cycle of $\mathrm{BSS}[0]$. If the three unknown bits are 0 (see line 3 in Fig. 8), signal sync is high at $t+7+4=t+11$. The counter is reset, and signal strobe is high at $t+11+3=t+14$. In the next cycle, the automaton reaches state $z^{t+15}=b[0]$. For any lower value of the counter, the automaton will reach this state earlier.

If the unknown bits are 1 , signal sync is high at $t+10+4=$ $t+14$. If the counter was 100 initially, then it has reached value 010 and strobe is high. At the same time, signal sync is high, the automaton stays in BSS[0] and the counter is reset. At cycle $t+17$, strobe is high and the automaton reaches $b[0]$ with a correct counter value at $t+18$. For any larger value, the automaton requires more cycles to reach this state.

From Theorem 4 and considering the possible values of $\beta_{c}^{\xi}$, we know for sure 6 bits of $\operatorname{BSS}[0]$ (from $t+1$ to $t+6$ ) and 6 bits of $\mathrm{BSS}[1]$ (from $t+9$ to $t+14$ ). Assuming that only these input values are known, the rest of the proof is purely digital. It is expressed by the following lemma, the proof of which is fully automatic.

\section{Lemma 2: From BSS[0] to BSS[1].}

$$
\begin{aligned}
& \quad \forall u \in[0: 6], i n p^{t+1+u}=1(* 6 \text { bits of BSS[1]*) } \\
& \wedge \quad \forall v \in[0: 6], i n p^{t+9+v}=0(* 6 \text { bits of BSS[0]*) } \\
& \wedge \quad\left(\left(z^{t}=\mathrm{BSS}[0] \wedge c n t^{t}=010 \vee 011\right)(* \text { states and *) }\right. \\
& \left.\vee \quad\left(z^{t}=\mathrm{FSS} \wedge c n t^{t}=001 \vee 010\right)\right)(* \text { misalignment*) } \\
& \rightarrow \quad \bigvee_{w \in[0: 3]} z^{t+15+w}=b[0] \wedge c n t^{t+15+w}=011 \\
& \text { Proof. By NuSMV. }
\end{aligned}
$$

\section{Strobing Correct Bits}

The next lemma states that whatever happens in the traversal of "synchronization" edges, strobe points always hit correct voted bits. We consider hypotheses similar to the previous lemma. The BSS[0]-mark matches the start point of the lemma. The automaton could be in state BSS[0] with counter at 011 or 100 , or in state FSS with counter at 001 or 010 . The reasoning is illustrated in the right part of Fig. 8, where voted bits are shown instead of the input.

Formally, we prove that register BYTE contains the correct frame 79 to 82 cycles following the first bit of the synchronization sequence. The proof shows the exact values of the counter that allow proper transmission. 


\section{RELATED WORK}

The first verification effort about physical layer protocols was carried out by Moore [11]. Moore developed a general model of asynchronous communications as a function in the logic of the ACL2 theorem prover [8]. Moore's model assumes distortion around sampling edges and do not allow for clock jitter. Sender and receiver modules are also represented by two functions. Moore's correctness criteria states that the composition of these three functions is an identity. He applied this approach to the verification of a Biphase-Mark protocol.

Moore's work inspired many studies around this protocol. Recently, Vaandrager and de Groot [17] modeled the protocol and analog behaviors using a network of timed-automata. Their model is slightly more general than Moore's and allows for clock jitter. They can derive tighter bounds for the BiphaseMark protocol. Previously, timed-automata have been used to verify a low level protocol based on Manchester encoding and developed by Philips [3]. Another recent proof of the BiphaseMark protocol has been proposed by Brown and Pike [4]. They developed a general model of asynchronous communications in the formalism of the tool SAL [10] developed at SRI. Their model includes clock jitter and metastability. Using $k$ induction, the verificaton of the parameterized specification of Brown and Pike is largely automatic. All these studies tackle protocol specification only. They prove functional correctness. We prove a more precise theorem about a gate-level hardware implementation and from which bounds on the transmission duration can be derived.

Regarding hardware verification, Hanna [6], [7] used predicates to approximate analog behaviors at the transistor level. The predicates can be embedded in digital proofs. His work is not specifically targeted to communication circuits and does not consider timing parameters, metastability or clock drift. We consider only gates and not their structure in terms of transistors.

\section{CONCLUSION AND FUtURE WORK}

Reliable transmission between two independent clocked devices is performed using bit clock synchronization, which is achieved by resetting a counter when detecting a synchronization sequence. This specific value is a crucial parameter. We have developed a general and precise model of asynchronous communications and defined a methodology to use this model for hardware design verification. We have proven the exact possible values for this parameter. This proves and disproves values proposed in the literature.

The model of clock domain crossing is about 2,000 lines and is available on the web ${ }^{3}$. The proof presented here was developed in about one man-year and is about 8,000 lines. Most of it is dedicated to the deduction of valid digital inputs from the analog transmission. This technique is independent of the design under verification. The analysis of similar designs will mainly amount to re-prove all digital lemmas.

\footnotetext{
${ }^{3}$ www.cs.ru.nl/ julien/, then Research
}

The case study is extracted from a more complex design which includes a scheduler implementing a high-level clock synchronization algorithm. We are currently applying our approach to the verification of this component, moving towards a fully verified distributed system.

\section{ACKNOWLEDGMENTS}

Tsverdyshev provided a precious technical support on the use of NuSMV within Isabelle. Shadrin wrote the translator from Isabelle to Verilog. This work initiates from the lecture "Computer Architecture 2 - Automotive Systems" given by Paul at Saarland University and notes taken by students ${ }^{4}$.

\section{REFERENCES}

[1] S. Beyer et al., Towards the Formal Verification of Lower System Layers in Automotive Systems, In Proc. of the 23rd IEEE International Conference on Computer Design (ICCD 2005), 2005.

[2] W. R. Bevier, W. A. Hunt Jr., J Strother Moore and W. D. Young, An Approach to Systems Verification, Journal of Automated Reasoning 5(4):411-428, 1989

[3] D. Bosscher, I. Polak and F. Vaandrager, Verification of an Audio Control Protocol, In Formal Techniques in Real-Time and Fault-Tolerant Systems (FTRTFT'94), pp. 170-192, LNCS 863, Spinger 1994

[4] G.M. Brown and L. Pike, Easy Parameterized Verification of Biphase Mark and 8N1 Protocols, In Proc. of 12th Intl. Conf. on Tools and the Construction of Algorithms (TACAS'06), pp. 58-72, LNCS 3920, Springer, 2006.

[5] FlexRay Communication System - Protocol Layer Specification v2.1, Rev A, FlexRay Consortium, December 2005.

[6] K. Hanna, Reasoning About Real Circuits, in Proc. of the 7th International Workshop on Higher Order Logic Theorem Proving and Its Applications, pp 235-253, Springer-Verlag, 1994

[7] K. Hanna, Automatic Verification of Mixed-Level Logic Circuits, In Proc. of the Second International Conference on Formal Methods in Computer-Aided Design (FMCAD '98), LNCS 1522, pp 133-166, Springer-Verlag 1998.

[8] M.Kaufmann, P.Manolios, and J.Moore, Computer Aided Reasoning: an Approach. Kluwer Academic Press, 2002.

[9] R. Männer, Metastable States in Asynchronous Digital Systems: Avoidable or Unavoidable ? Microelectronics Reliability, 28(2):295-307, 1988

[10] L. de Moura et al., SAL 2, In Computer-Aided Verification (CAV'04), pp. 496-500, LNCS 3114, Springer, 2004.

[11] J Strother Moore, A Formal Model of Asynchronous Communications and Its Use in Mechanically Verifying a Biphase Mark Protocol, Formal Aspects of Computing,6(1) 60-91, 1993.

[12] J Strother Moore, A Grand Challenge Proposal for Formal Methods: A Verified Stack, In $10^{\text {th }}$ Anniversary Colloquium of UNI/IIST, B. K. Aichernig and T. S. E. Maibaum editors, LNCS 2757, pp 161-171, Springer, 2003.

[13] T. Nipkow, L.C. Paulson and M. Wenzel, Isabelle/HOL: A Proof Assistant for Higher-Order Logic, Vol. 2283 of LNCS, Springer, 2002.

[14] V. Sagdeo, The Complete Verilog Book, Springer, 1998

[15] J. Schmaltz, A Formal Model of Lower System Layer, In Proc. of Formal Methods in Computer-Aided Design (FMCAD'06), San Jose, CA, USA, November 12-16, pp 191-192, IEEE/ACM, 2006.

[16] S. Tverdyshev, Combination of Isabelle/HOL with Automatic Tools. In Proc. of 5th International Workshop on Frontiers of Combining Systems, FroCoS 2005, LNCS 3717, pp 302-309, Vienna, Austria, September 19$21,2005$.

[17] F.W. Vaandrager and A.L. de Groot, Analysis of a Biphase Mark Protocol with UPAAL and PVS, Formal Aspects of Computing 18(4):433-458, Springer, 2006.

\footnotetext{
${ }^{4}$ www-wjp.cs.uni-sb.de/lehre/lehre.php
} 